\title{
EFFECTS OF THE OSCNODE UPON COVARIANT FORMS OF THE RATIONAL PLANE QUARTIC CURVE*
}

BY J. H. NEELLEY

1. Introduction. A recent paper $\dagger$ discusses some effects of the tacnode and ramphoid cusp upon the combinants of two line sections and other associated forms of the rational plane quartic curve. This paper observes those forms for the curve with an oscnode. Well known results have been purposely omitted.

2. The Ordinary Oscnode. As previously shown $\ddagger$ the oscnodal parameters may be taken as $t= \pm a$ if the two tangents of the pencil of lines on the oscnode have contacts at $t=0$ and $t=\infty$. These tangents and the join of their contacts give the curve the representation

$$
\left\{\begin{array}{l}
x_{0}=t^{4}-a^{2} t^{2}, \\
x_{1}=t^{2}-a^{2}, \\
x_{2}=4 t^{3}-2 \sigma t^{2}-4 a^{2} t,
\end{array}\right.
$$

where $\sigma=t_{1}-a^{2} t_{1}^{-1}$, the sum of the parameters of the points of contact of the distinct double line. Two line sections $(\xi x)=0,(\eta x)=0$ have combinants which are converted into covariant curves of $R_{2}^{4}$ by means of the relations

$$
\left|\begin{array}{cc}
(a \xi) & (b \xi) \\
(a \eta) & (b \eta)
\end{array}\right|=|a b x|,
$$

and so forth, where the third-order determinants are formed from equations (1). $\S$ The discriminant of the invariant

* Presented to the Society, September 6, 1928.

$\dagger$ Neelley, Concerning covariants of the rational plane quartic curve with compound singularities, this Bulletin, vol. 35, pp. 67-72.

$\ddagger$ Neelley, Compound singularities etc., American Journal of Mathematics, vol. 49 , p. 397 .

$\S$ Rowe, Transactions of this Society, vol. 12, p. 296. 
$\overline{12}^{4}(1,1)$ of the pencil $(\xi x)+\lambda(\eta x)=0$ is the quadratic form which becomes the conic $g_{2},{ }^{*}$ the envelope of the self-apolar line sections of the curve. The condition that the quartics cut out by $(\xi x)$ and $(\eta x)$ be derived functions of a quintic is another second-order combinant which becomes the Stahl conic $K, \dagger$ the conic on the vertices of the flex-triangles of the first osculants of $R_{2}{ }^{4}$. The pencil $g_{2}+\lambda k=0$ is used to study the effects of the singularity with only well known results for this type of the curve.

The fundamental involution is determined by the quartics

(2) $(\beta t)^{4} \equiv 4 t^{3}+4 a^{2} t, \quad(\gamma t)^{4} \equiv t^{4}+2 \sigma t+6 a^{2} t^{2}+a^{4}$.

The complete system of covariants of these two quartics has the $g_{3}$ of the first quartic and $\overline{1} \overline{2}^{4}\left(1, H_{2}\right)$ and $\overline{1} \overline{2}^{4}\left(2, H_{1}\right)$ identically zero. We also note that the form $\overline{1}^{2}(1,2)$ is a quartic with the same roots as those of the quartic cut from the curve by the reference line $x_{0}=0$. We shall observe this form for the special cases of the singularity.

3. The Oscnodal Cusp. This type of the oscnode assumes the properties of the ramphoid cusp as well as those resulting from the coincidence of three adjacent nodes. If the nodal parameter is $t=\infty$ and the contact of the distinct (improper) double line on the node is $t=0$, the nodal line, this double line, and a line on $t=0$ can be taken so as to represent the curve by $\ddagger$

$$
\left\{\begin{array}{l}
x_{0}=e, \\
x_{1}=6 c t^{2}, \\
x_{2}=12 a t^{4}+12 d t,
\end{array}\right.
$$

with

$$
(\beta t)^{4} \equiv 4 a t, \quad(\gamma t)^{4} \equiv 4 a t^{3}+d .
$$

* Rowe, loc. cit., pp. 298-299; Salmon, Modern Higher Algebra, 4th edition, p. 219.

$\dagger$ Stahl, Journal für Mathematik, vol. 101, pp. 300-325.

¥Winger, American Journal of Mathematics, vol. 36, p. 65. 
Consideration of the combinants of $(\xi x)$ and $(\eta x)$ gives the conics $g_{2}$ and $K$ as

$$
a e x_{1}^{2}+c^{2} x_{0} x_{2}=0
$$

and

$$
c x_{0} x_{2}=0
$$

respectively. So $K$ reduces to two sides of the triangle of reference, the oscnodal line being one. Obviously the pencil of conics $g_{2}+\lambda K$ is a double-contact pencil and the conics of the pencil have contact with $R_{2}{ }^{4}$ at the cusp. The conic on the flexes and the points $q_{i}{ }^{*}$ is of this pencil and has the equation

$$
3 a e x_{1}^{2}-c^{2} x_{0} x_{2}=0 .
$$

As this is on the point $(1,0,0)$ and as it has previously been shown that three flexes and one point $q$ coincide at a ramphoid cusp $\dagger$ we have the following theorem.

THEOREM 1. The oscnodal rational plane quartic curve with a cusp has its points $q_{i}$ on the distinct double line.

But more interesting is the fact that the general equation of the Stahl conic $N \ddagger$ reduces to the square of this distinct double line. Now the conic $N$ may be defined as the locus of a point from which a pencil of lines will cut a pencil of quartics from the curve $R_{2}^{4}$ for which the invariant $I_{2}^{\prime}$ vanishes. The line obviously satisfies such a condition as. any line $(\lambda x)=0$ and $x_{1}=0$ give such a pencil of quartics. We have therefore the following result.

THEOREM 2. The conic $N$ meets the curve in two pairs of coincident points, the points $q_{i}$, when the oscnode is a cusp.

The covariant system of the fundamental quartics (4) has several forms which vanish identically. The quartics

* Rowe, Transactions of this Society, vol. 13, p. 390.

$\dagger$ Neelley, Compound singularities etc., American Journal of Mathematic, vol. 49 , p. 390 .

$\ddagger$ Stahl, Journal für Mathematik, vol. 101, pp. 300-325. 
are self-apolar and each is apolar to the Hessian of the other. This is characteristic of the oscnodal cusp. The ramphoid cusp covariant, the quadratic resulting from operating with the first of the quartics upon the Jacobian of the second and its Hessian, ${ }^{*}$ vanishes here also. Then the quartic $\overline{1}^{2}(1,2)$ is the same as that cut out by the line $x_{1}=0$. So again this form is a line section of the curve. The covariant forms of this system are very degenerate and many syzygetic relations exist.

4. The Knot. This special form of the oscnode is such that any line on the singular point meets the curve in three coincident points and the oscnodal line meets it in four, all with the same parameter value. The oscnodal line at $t=0$ and the line on the oscnode and the point $t=\infty$ with the tangent at the latter point give $R_{2}^{4}$ as follows:

$$
\left\{\begin{array}{l}
x_{0}=a t^{4}, \\
x_{1}=4 b t^{3}, \\
x_{2}=6 c t^{2}+4 d t+e,
\end{array}\right.
$$

with

$$
(\beta t)^{4} \equiv d t^{4}+e t^{3}, \quad(\gamma t)^{4} \equiv c t^{3}+d t^{2} .
$$

The conic $g_{2}$ has the equation

$$
\left(4 b d x_{0}+a e x_{1}\right)^{2}=0,
$$

which is the square of a line on the oscnode. The conic $K$ is the square of the oscnodal line

$$
x_{0}^{2}=0 .
$$

So the pencil $g_{2}+\lambda K$ is a degenerate one with its vertex at the oscnode. The conic $K$ has all of its intersections with $R_{2}{ }^{4}$ at $t=0$ and since six of those of $g_{2}$ and the curve are at the same point the conic on the flexes has at least six intersections with $R_{2}^{4}$ at the oscnode. Now the points $q_{i}$ are given by $\overline{1} 2^{3}(1,2)$ of the system of the quartics (9) and this form

\footnotetext{
* Neelley, Concerning covariants etc., this Bulletin, vol. 35, p. 72.
} 
is the quadratic $t^{2}=0$. Hence we have the following theorem.

THEOREM 3. The two points $q_{2}$ coincide at the knot on the oscnodal rational plane quartic curve.

The resultant of the line sections is the very simple equation of the curve given below:

(12) $a^{3} e x_{1}^{4}+16 a^{2} b d x_{0} x_{1}^{3}+96 a b^{2} c x_{0}^{2} x_{1}^{2}-256 b^{4} x_{0}{ }^{3} x_{2}=0$.

Again the forms of the system of the fundamental quartics are in many cases dependent. Many relations vanish identically; all of the independent quadratic forms do except $\overline{12}^{3}(1,2)$ and $\overline{12}^{3}\left(2, H_{1}\right)$. Once more we find that $\overline{1}^{2}(1,2)$ is apolar to each of the fundamental quartics and is consequently a line section. When formed for types of the curve without an oscnode $\overline{12}^{2}(1,2)$ is not a line section, but it has been shown to be for all types with the oscnode, so we suggest the following theorem.

TheOREM 4. The quartic form $\overline{12^{2}}(1,2)$ of the system of the fundamental quartics of the rational plane quartic curve locates four points on a line if the curve has an oscnode, the line being on the oscnode.

Carnegie Institute of Technology 